\title{
Erythrocyte sedimentation rate in diabetic and non-diabetic patients of cardiovascular disease
}

\author{
Mubin Mustafa Kiyani ${ }^{1 a}$, Saira Jahan ${ }^{2 b}$, Sania Khawar Kiani ${ }^{1 c}$, Hamza Rehman ${ }^{3 d}$, Lal Gul Khan ${ }^{1 e}$, Umar \\ |qbal ${ }^{1 \mathrm{f}}$
}

${ }^{1}$ Faculty of Rehabilitation and Allied Health Sciences, Riphah International University, Islamabad, Pakistan

${ }^{2}$ Department of Biochemistry, Islamic International Medical College, Riphah International University, Islamabad, Pakistan

${ }^{3}$ Department of Bioinformatics and Biotechnology, Faculty of Basic and Applied Sciences, International Islamic University, Islamabad, Pakistan

aEmail address: mubin3us@yahoo.com

bEmail address: docsaraiimc@gmail.com

cEmail address: sania.khawar@riphah.edu.pk

dEmail address: hamzarehman51@gmail.com

eEmail address: lalgul.khan@riphah.edu.pk

‘Email address: umar.iqbal@riphah.edu.pk

\section{HIGHLIGHTS}

The elevated level of ESR in CVD patients might indicate the prognosis of diabetes mellitus

\section{ARTICLE INFO}

\section{Article history}

Received date: March $24^{\text {th }}, 2019$

Revised date: Mei $03^{\text {rd }}, 2019$

Accepted date: Mei 03 ${ }^{\text {rd }}, 2019$

\section{Keywords:}

Diabetes mellitus

Erythrocyte sedimentation

Cardiovascular diseases

\begin{abstract}
A B S T R A C T
The pervasiveness of global endemic of type II diabetes, driven by population development, increasing obesity, urbanization, and other additional unidentified factors may be crumpled in the next 20yrs. The purpose of this study was to determine the correlation of ESR in diabetic and non-diabetic patients of cardiovascular diseases. It is a co-relational study conducted at KRL hospital Islamabad from February 2018 to September 2018. The study population compromised a total of 180 cases aged 45 to 50 years were taken, in which 60 cases of CVD with DM, 60 cases of CVD without DM and 60 normal healthy population were taken. All patients and controls gave written informed consent. All subjects went through a general physical examination, and a questionnaire was used to collect the records about demographics and past medical history and existing use of medications. In the presented study, Pearson's Correlation between ESR of healthy and ESR of CVD without DM shows a weak correlation between these two variables i.e., 0.127 with a $p$-value of 0.503 . We also establish that there is a direct intermediate relationship between ESR of healthy and ESR of CVD with DM. High level of ESR in CVD patients might indicate the prognosis of DM. To confirm this further researches and studies must be conducted in this area.
\end{abstract}

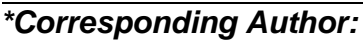 \\ Mubin Mustafa Kiyani \\ Faculty of Rehabilitation and Allied Health Sciences \\ Riphah International University, Islamabad, Pakistan \\ Email:mubin3us@yahoo.com
}




\section{INTRODUCTION}

The pervasiveness of global endemic of type II diabetes, driven by population development, increasing obesity, urbanization, and other additional unidentified factors may be crumpled in the next 20yrs. ${ }^{1}$ India, the world's second most crowded country, has made an astounding economic development in recent years but on the other hand however, currently it treasures itself lagging behind peers on healthcare consequences. Every fifth person in India is diabetic, and because of this, it made India the world's capital of Diabetics so this disease is pretentiousness a massive health issues in the country. ${ }^{2}$, ${ }^{3}$ Whereas, the pervasiveness of diabetes in the urban compared to the rural areas of Pakistan was $6.0 \%$ in men and $3.5 \%$ in women while $6.9 \%$ in men and $2.5 \%$ in women, correspondingly. ${ }^{4}$

On the whole glucose intolerance diabetes mellitus and impaired glucose tolerance (DM+IGT) was $22.04 \%$ in urban and $17.15 \%$ in rural areas of Pakistan. ${ }^{4}$ At present, Pakistan defenses $6^{\text {th }}$ between the countries with the whole load of diabetes mellitus. ${ }^{1}$ In the previous 50 years, health care systems of the world have faced a new epidemic twofold disease: cardiovascular diseases and diabetes mellitus. At present, CVD is the foremost root of death in all western countries, and $60 \%$ of deaths for ischemic heart disease and stroke takes place in developing countries with setting up possessions, and the conspicuous relationship between peripheral artery disease, stroke, coronary artery disease, and diabetes have enforced physicians to explore the probable pathophysiological association amongst these dissimilar clinical circumstances. Latest and persuasive evidence has revealed the noteworthy and self-regulating role of inflammation, insulin resistance and subsequent endothelial dysfunction in the commencement and succession of atherothrombosis, superimposed on conventional risk factors. ${ }^{5}$

The importance of the erythrocyte sedimentation rate is also examined in the background of heart failure, even though previous studies recommended a poorer prognosis of chronic heart failure with low down ESR. ${ }^{6}$ Current studies on patients with chronic heart failure treated with angiotensin-converting enzyme inhibitors showed that an elevated ESR might be an adverse prognostic indication, independent of patients' symptomatology and ventricular function. ESR is also positively associated with proinflammatory cytokines, ${ }^{6,7}$ that have been revealed to forecast heart failure even in the asymptomatic populace, and that appears to be linked with worse prognosis in patients with heart failure. ${ }^{8,9}$ In recent years, the relationship between inflammation and cardiovascular diseases has gained significant interest. ${ }^{10}$ Numerous systemic markers of inflammation, together with an erythrocyte sedimentation rate (ESR), have been established to be predictors of coronary heart disease. ${ }^{11} \mathrm{C}$-reactive protein, interleukin (IL)-6, and tumor necrosis factor (TNF)-alpha and all markers of cytokine-mediated inflammation, have been revealed to predict occurrence heart failure (HF). ${ }^{12}$ Erythrocyte sedimentation rate, a wide-ranging indicator of inflammation an included measure of acute stage response is enlarged in several inflammatory circumstances. ${ }^{13}$ Erythrocyte sedimentation rate is an extensively used, frequently executed, and low-priced laboratory test to find out the red blood cell accumulation. ESR in a diabetic patient may be raised in the nonexistence of unconcealed infection. ${ }^{14}$ The purpose of the present study was to determine the correlation of ESR in diabetic and non-diabetic patients of cardiovascular diseases.

\section{MATERIALS AND METHOD}

This research performed a correlational study from February 2018 to September 2018, assessing the level of ESR in 60 stable patients of CVD without DM and 60 stable patients of CVD with DM from KRL Hospital Islamabad. ESR measurement was also done in 60 healthy subjects. All patients and controls gave written informed consent. 
All subjects went through a general physical examination, and a questionnaire was used to gather the records concerning demographics and past medical history and existing use of medications. The diagnosis of CVD and DM was based on clinical presentation, standard investigations, and examination. Diabetes occurrence data for adults aged 45 to 50 years were taken. Patients were disqualified from the study if they were clinically unstable, were edematous, had dyspnea, or had severe renal failure. No subject had clinical signs of infection, rheumatoid arthritis, or cancer at the time of ESR measurement.

The ESR was determined by using the Westergren method. In brief, $4.5 \mathrm{~mL}$ of venous blood was drawn into a tube containing $3.8 \%$ solution of sodium citrate. The test tube was mixed gently right away following blood sampling and again just before measuring, which took place within 2 hours after the blood was drained. A standard 200$\mathrm{mm}$, Westergren method glass tube was filled to the zero marks at the top, placed in a vertical position, and left for 1 hour. The distance from the bottom of the surface of the tube to the top of the column was then calculated, and the result was articulated as millimeters in the first hour. The blood samples were then analyzed for ESR, total cholesterol, high and low-density lipoprotein cholesterol (HDL-C and LDL-C), plasma triglycerides, and homocysteine. Data were analyzed by using SPSS 21.

\section{RESULTS AND DISCUSSION}

The relationship between erythrocyte sedimentation rate (ESR) in diabetic (DM) and non-diabetic patients of cardiovascular diseases (CVD) were investigated in a total of 180 participants in which 60 patients of cardiovascular diseases with diabetes in group A, 60 patients of cardiovascular diseases without diabetes in group $B$ and 60 healthy participants were included. ESR Strength of CVD with DM, CVD without DM and with normal healthy population was determined by using Pearson correlation ' $r$ ' of the patients included in the study, $116(64 \%)$ were male with the mean age was $61.8 \pm 10.3$ years and $64(36 \%)$ were females with the mean age was $57.7 \pm 9.5$ years. The mean values of ESR among these three groups were $35.23+5.20,33.03+4.98$ and $17.20+1.73$, $33.03+4.98 \mathrm{~mm} / \mathrm{hr}$ respectively.

Pearson's Correlation ' $r$ ' between ESR of healthy and ESR of CVD without DM shows a weak correlation between these two variables i.e., 0.127 with a $p$-value of 0.503 , as shown in Table 1. This result means that changing in one variable is weakly correlated with changing in the second variable. In our result, the Pearson Correlation ' $r$ ' is 0.127 , and no clear association establishes between ESR of healthy and ESR of CVD patients without DM. In the present study, we also determine that there is a direct intermediate relationship between ESR of healthy and ESR of Cardiovascular disease with diabetic patients. In this result, the Pearson ' $r$ ' is 0.228 with a $p$-value of 0.225 , as shown in table 01 . So, there is no clear association or interaction between the ESR of healthy and ESR of CVD with DM patients. We also calculate Pearson correlation between ESR of CVD without DM and ESR of CVD with DM, and we determined that there is also a weak relationship between these two variables. Value of Pearson ' $r$ ' between these two variables is 0.160 (see table 01). Probably, elevated ESR, CVD, and hyperglycemia are associated with worse prognosis by a different mechanism.

Results of comparing ESR of healthy samples with those of CVD patients without DM did not show anything to ponder on regarding ESR. Same was the case with healthy samples and diabetic patients with cardiovascular diseases. 
Table 1. Correlations between ESR of healthy, ESR of CVD without DM and ESR of CVD with DM.

\begin{tabular}{llll}
\hline & $\begin{array}{c}\text { ESR of } \\
\text { healthy }\end{array}$ & ESR of CVD without DM & ESR of CVD with DM \\
\hline Pearson correlation & & .127 & .228 \\
$P$ value & & .503 & .225 \\
Pearson correlation & .228 & .160 & \\
$P$ value & .225 & .399 & \\
\hline
\end{tabular}

$\mathrm{ESR}=$ Erythrocyte sedimentation rate, $\mathrm{CVD}=$ cardiovascular diseases, $\mathrm{DM}=\mathrm{Diabetes}$ mellitus

The previous study based on inflammation measured by ESR and development of heart failure stated that ESR is an independent predictor of $\mathrm{HF}^{15}$; similarly, another research was done to find a relation between ESR and micro-inflammation, that research concluded that increased or decreased ESR is easy means to detect microinflammation. ${ }^{16}$ Another research was done finding a relation between ESR and myocardial infarction, and according to the conclusions of that research, there is an association between raised ESR and long-term mortality in ST-elevated Myocardial infarction patients. ${ }^{17}$ ESR and heart's connection was also tested by another study carried out based on a relation between ESR, atherosclerosis and cardiac mortality and result of that study also avers that ESR is an independent indicator of coronary atherosclerosis. ${ }^{18}$

Following previous studies, it was considered vital to determine the role of ESR in CVD with and without DM. So, this study was carried out to ascertain the role of ESR in cardiovascular disease and diabetes mellitus. After calculating ESR from samples of healthy people, those having CVD with DM and people having CVD without DM, results were compared and analyzed to find an association between them regarding ESR as a determinant of inflammation. When the results of the ESR of healthy and ESR of CVD patients without DM were compared, it was discerned that there is no lucid association between them. This means that no matter what ESR is, it has no significant effect on the onset of CVD without DM or its progress.

While comparing Pearson ' $r$ ' with $p$ values from samples of healthy and CVD patients with DM it was observed that there is no link or significant association between the ESR of them, means that there is also no noted association between ESR and CVD with DM. It was earlier thought that the same might be the case with ESR and CVD with DM, but results nullified this notion. Another study regarding ESR and coronary heart disease says that further studies and research is needed on ESR regarding its role in heart diseases. ${ }^{19}$ Following those results, it was considered a probability that ESR might also play a role in CVD patients with and without DM. Now ESR of CVD patients with DM and CVD patients without DM were compared. Results after were somehow different. It showed a weak relationship between variables and elevated ESR, CVD, and hyperglycemia are associated. That meant that the increase in ESR in CVD patients might indicate the prognosis of DM.

\section{CONCLUSION}

High level of ESR in CVD patients might indicate the prognosis of diabetes mellitus. To confirm this further researches and studies must be conducted in this area. If this is so, it can be beneficiary for CVD patients to detect their risk of developing DM by ESR, which is an inexpensive laboratory test.

\section{REFERENCE}

1. Wild, S., et al., Global prevalence of diabetes estimates for the year 2000 and projections for 2030. Diabetes Care, 2004. 27(5): p. 1047-1053. 
2. Swami, S.S., et al., Hypertension and diabetes in India: a review. Int J Clin Biochem Res, 2015. 2(March): p. 54-8.

3. Joshi, S.R. and R.M. Parikh, India; The Diabetes Capital of the World: Now heading Towards Hypertension. Journal-Association Of Physicians Of India, 2007. 55(Y): p. 323.

4. Shera, A., F. Jawad, and A. Maqsood, Prevalence of diabetes in Pakistan. Diabetes research and clinical practice, 2007. 76(2): p. 219-222.

5. Cosentino, F., and G. Egidy Assenza, Diabetes, and inflammation. Herz, 2004. 29(8): p. 749-759.

6. Sharma, R., et al., The relationship of the erythrocyte sedimentation rate to inflammatory cytokines and survival in patients with chronic heart failure treated with angiotensin-converting enzyme inhibitors. Journal of the American College of Cardiology, 2000. 36(2): p. 523-528.

7. Bozkurt, B., D.L. Mann, and A. Deswal, Biomarkers of inflammation in heart failure. Heart failure reviews, 2010. 15(4): p. 331-341.

8. Maradit-Kremers, $\mathrm{H}_{\text {., }}$ et al., Raised Erythrocyte Sedimentation rate signals heart failure in patients with rheumatoid arthritis. Annals of the rheumatic diseases, 2007. 66(1): p. 76-80.

9. Deswal, A., et al., Cytokines and cytokine receptors in advanced heart failure an analysis of the cytokine database from the Vesnarinone Trial (VEST). Circulation, 2001. 103(16): p. 2055-2059.

10. Willerson, J.T., and P.M. Ridker, Inflammation as a cardiovascular risk factor. Circulation, 2004. 109(21 suppl 1): p. II-2-II-10.

11. Danesh, J., et al., C-reactive protein and other circulating markers of inflammation in the prediction of coronary heart disease. New England Journal of Medicine, 2004. 350(14): p. 1387-1397.

12. Cesari, M., et al., Inflammatory markers and onset of cardiovascular events results from the Health ABC Study. Circulation, 2003. 108(19): p. 2317-2322.

13. Sudha, R., et al., Serum Sialic Acid in Relation to Erythrocyte Sedimentation Rate And HBA of Type 2 Diabetic Patients.

14. Music, M., et al., Analysis of Inflammatory parameters in Diabetes Mellitus Type 2 patients with or without Metabolic Syndrome. Materia Socio-Medica, 2010. 22(2): p. 64.

15. Ingelsson, E., et al., Inflammation, as measured by the erythrocyte sedimentation rate, is an independent predictor for the development of heart failure. Journal of the American College of Cardiology, 2005. 45(11): p. 1802-1806.

16. Steinvil, A., et al., Determinants of the Erythrocyte Sedimentation Rate in the Era of Microinflammation. American journal of clinical pathology, 2008. 129(3): p. 486491.

17. Timmer, J., et al., Prognostic value of erythrocyte sedimentation rate in ST segment elevation myocardial infarction: interaction with hyperglycemia. Journal of internal medicine, 2005. 257(5): p. 423-429.

18. Natali, A., A. L'Abbate, and E. Ferrannini, Erythrocyte sedimentation rate, coronary atherosclerosis, and cardiac mortality. European heart journal, 2003. 24(7): p. 639648.

19. Gillum, R.F., M.E. Mussolino, and D.M. Makuc, Erythrocyte sedimentation rate and coronary heart disease: the NHANES I Epidemiologic Follow-up Study. Journal of clinical epidemiology, 1995. 48(3): p. 353-361. 